Check for updates

Cite this: J. Mater. Chem. A, 2019, 7, 1149

Received 23rd November 2018 Accepted 6th December 2018

DOI: $10.1039 / c 8 t a 11298 c$

rsc.li/materials-a

\section{Concave curvature facets benefit oxygen electroreduction catalysis on octahedral shaped PtNi nanocatalysts $\uparrow$}

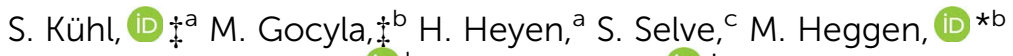 \\ R. E. Dunin-Borkowski iD ${ }^{b}$ and P. Strasser iD *a
}

Studies that demonstrated enhanced electrocatalytic oxygen reduction activities of octahedral PtNi nanocatalysts have routinely motivated and explained their data by the structure-sensitivity of PtNi alloy surfaces in general, more specifically by the favourable performance of the annealed $\mathrm{Pt}_{3} \mathrm{Ni}(111)$ single crystal surface with a monoatomic Pt skin, in particular. In this contribution, we challenge this view and show that imperfect Ni-enriched $\{111\}$ nanofacets with concave Pt curvature catalytically outperform flat, well-alloyed, locally ordered $\{111\}$ and $\{100\}$ nanofacets in cuboctahedral nanoparticles. To achieve this, we investigate the geometric, compositional, and morphological structure on the ensemble and on the individual particle level of PtNi alloy nano-octahedra. In particular, we track the correlations of these parameters after thermal annealing and link them to their catalytic activity. The level of local compositional and structural disorder appears to be a reliable descriptor and predictor for ORR activity at least within a family of catalysts. After annealing up to $300{ }^{\circ} \mathrm{C}$, concave Pt $\{111\}$ facets, with partially flat $\mathrm{Ni}$ facets, remained most prevalent, resulting in nanoparticles with pronounced elemental anisotropy. At higher annealing temperature, concave Pt morphologies gave way to cuboctahedra with healed flat $\{111\}$ and $\{100\}$ alloy facets. The imperfect concave nano-octahedral catalysts with enhanced local disorder invariably outperformed more ordered particles, yet lagged behind in morphological stability. Faceted PtNi nano-cuboctahedra emerging at $400{ }^{\circ} \mathrm{C}$ ultimately offered the most reasonable balance between moderately high activity and good morphological stability. This is why we propose these cuboctahedral shaped Pt alloy nanoparticles as promising PEM cathode fuel cell catalyst of choice. While the present results do not invalidate the exceptional oxygen reduction activity of perfect $\mathrm{Pt}_{3} \mathrm{Ni}(111)$ "skin" single crystal surfaces, they shed new light on the decade old puzzle about structure-activity relationships of PtNi octahedral nanocrystals.

\section{Introduction}

Single crystal studies have revealed that the oxygen reduction reaction (ORR) on platinum surfaces is a highly structuresensitive electrocatalytic reaction. ${ }^{\mathbf{1} 2}$ The trend in ORR activity of low-index pure Pt single crystal surfaces in non-adsorbing electrolytes follows the order $\operatorname{Pt}(100) \ll \operatorname{Pt}(111) \approx \operatorname{Pt}(110) .^{3,4}$

\footnotetext{
${ }^{a}$ Department of Chemistry, Chemical Engineering Division, Technical University Berlin, 10623 Berlin, Germany. E-mail: pstrasser@tu-berlin.de

${ }^{b}$ Ernst Ruska-Centre for Microscopy and Spectroscopy with Electrons, Forschungszentrum Jülich GmbH, 52425 Jülich, Germany. E-mail: m.heggen@ fz-juelich.de

'ZELMI - Central Facility for Electron Microscopy, Technical University Berlin, 10623 Berlin, Germany

$\dagger$ Electronic supplementary information (ESI) available: ICP and XRD results including parameters of Rietveld refinement, TEM particle size distributions and additional results from electrochemical testing, and additional TEM images after electrochemical testing. See DOI: 10.1039/c8ta11298c

\$ These authors contributed equally to the article.
}

Likewise, Pt alloy surfaces have demonstrated a sensitive dependence of their catalytic ORR activity on their surface atomic arrangement. ${ }^{4}$ Most notably, a $\mathrm{Pt}_{3} \mathrm{Ni}(111)$ single crystal facet, prepared in a $700{ }^{\circ} \mathrm{C}$ annealing process during which a single atom Pt "skin" layer segregated to the top of the surface, exhibited a 10-fold activity improvement over a Pt(111) single crystal facet. ${ }^{4}$ In the wake of this report, during the last decade, a large number of studies focused on the development of suitable syntheses of octahedral-shaped PtNi nanoparticles (NPs) with a sufficiently high number of $\{111\}$ surfaces to enhance both specific and mass activity. ${ }^{5-9}$ Even though all of these studies on octahedral PtNi nanocatalysts closely refer to the ideal $\mathrm{Pt}_{3} \mathrm{Ni}(111)$ single crystal surface with a Pt skin, not even one of these studies actually succeeded in preparing the intended PtNi alloy nano-octahedra with exclusive exposure of $\{111\}$-oriented Pt skin layer facets. This is most likely because none of the reported synthetic routes toward nano-octahedra involved thermal annealing at sufficiently high temperatures, where the thermal segregation of a Pt monolayer (the Pt skin) on 
top of a well-alloyed PtNi crystal would occur. On the other hand, application of a temperature of $700{ }^{\circ} \mathrm{C}$, at which this process takes place, would invariably destroy the octahedral shape $^{\mathbf{1 0}}$ and, hence, the prevalence of $\{111\}$ facets. Nonetheless, most of the reported octahedral nanocatalysts featured electrocatalytic ORR activity benefits in line or even greatly exceeding the original 10-fold improvement over pure Pt of the structurally well-defined $\mathrm{Pt}_{3} \mathrm{Ni}(111)$ single crystal surface with a Pt skin structure. Evidently, the well-ordered $\mathrm{Pt}_{3} \mathrm{Ni}(111) \mathrm{Pt}$ skin surface with its unique electronic and structural arrangements is not the only type of PtNi alloy surface that is able to efficiently catalyze the electroreduction of oxygen. ${ }^{\mathbf{1 1}}$ Actually, as reported recently, other beneficial chemical or structural factors previously overlooked appear to weigh in as well. ${ }^{\mathbf{1 2}}$

In the present work, we study the influence of the local atomic structural and compositional order of facets of Ni-rich PtNi nano-octahedra on their electrocatalytic ORR activity. We compare nano-octahedral facets featuring a Pt-concave $\{111\}$ morphology with compositionally homogeneous and structurally more ideal, flat $\{111\}$ and $\{100\}$ nano-cuboctahedral facets. To achieve this, we analyse the structure, and image and element-map the morphology and composition of PtNi nanoparticles after thermal reductive annealing, and link them to their ORR activity and stability during potential cycling. We argue that like all previous studies, at no point in time, the atomic surface structure of our own nano-octahedra resembles even remotely the originally reported ideal $\mathrm{Pt}_{3} \mathrm{Ni}(111)$ structure with a "Pt skin" overlayer. However, we provide clear evidence that the Pt-enriched, concave $\{111\}$ facets unfold a highly beneficial ORR activity. In contrast, well-annealed smooth alloy facets perform catalytically less active. To explain this finding, we report and discuss a strong correlation between Pt-concave $\{111\}$ facets and the presence of local microstrain that appears to be a reliable experimentally accessible descriptor for the catalytic activity of PtNi nano-alloy particles. We ultimately conclude that the quest for an ideally ordered $\mathrm{Pt}_{3} \mathrm{Ni}$ nanooctahedron with the original Pt skin structure may be, albeit very challenging, worth pursuing. Our findings and conclusions show that catalytically equally or even more active nano-alloy facets can be designed using Pt-concave $\{111\}$ facets. In other words, stepped and highly rough surfaces outperform ideally ordered ones in catalytic performance and tend to be synthetically more accessible.

\section{Experimental}

\section{Synthesis}

For the synthesis of octahedral PtNi nanoparticles, $0.2 \mathrm{mmol}$ $\mathrm{Pt}(\text { acac })_{2}$ and $\left.1.4 \mathrm{mmol} \mathrm{Ni(acac}\right)_{2}$ were dissolved in $50 \mathrm{~mL} \mathrm{DMF}$ by ultrasonication for $5 \mathrm{~min}$. The homogeneous solution was transferred into a glass-lined stainless steel autoclave. The sealed autoclave was heated from room temperature to $130{ }^{\circ} \mathrm{C}$ within $30 \mathrm{~min}$. The temperature was held for $42 \mathrm{~h}$ before it was naturally cooled down to room temperature. Afterwards the particles were supported on carbon (Vulcan XC72R) by $5 \mathrm{~min}$ ultrasonication and under around $23 \mathrm{~h}$ static conditions at room temperature. Finally, the PtNi/C was washed with ethanol/ water several times and freeze-dried afterwards. This synthesis is based on that reported by Cui et al. ${ }^{9}$

For annealing, a furnace tube with a portion of the asprepared sample was purged with nitrogen for $1 \mathrm{~h}$ at room temperature. Afterwards, the atmosphere was changed to $4 \%$ $\mathrm{H}_{2} / \mathrm{Ar}$ and the temperature was raised at $10 \mathrm{~K} \mathrm{~min}^{-1}$ to a temperature between 200 and $500{ }^{\circ} \mathrm{C}$, and held for $30 \mathrm{~min}$. Afterwards it was cooled down in a nitrogen flow to room temperature. The obtained samples are labeled " $\mathrm{H}_{2} \mathrm{~T}$ " with $T$ being the annealing temperature.

\section{Physical and chemical characterization}

Inductively coupled plasma-optical emission spectroscopy (ICPOES) was used for elemental and compositional analysis using a Varian 715-ES spectrometer with a CCD detector. Samples were digested in acid $\left(\mathrm{HNO}_{3}: \mathrm{H}_{2} \mathrm{SO}_{4}: \mathrm{HCl}=1: 1: 3\right)$ and heated using microwave irradiation to $180{ }^{\circ} \mathrm{C}$ for $10 \mathrm{~min}$, with a ramping step of $10 \mathrm{~min}$. The samples were diluted with Milli-Q water $\left(>18 \mathrm{M} \Omega \mathrm{cm}^{-1}\right)$ to reach appropriate emission intensity. Standards with a known concentration were co-analysed with the samples.

Powder X-ray diffraction (XRD) was carried out on a D8 Advance X-ray diffractometer (Bruker AXS) working with a position sensitive LynxEye detector (PSD) and a $\mathrm{Cu} \mathrm{K}_{\alpha}$ source. The diffraction patterns of the catalysts were recorded at diffraction angles between 20 and $90^{\circ}$ with a step size of $0.05^{\circ}$ and a collection time of $10 \mathrm{~s}$. Rietveld refinement was performed with Topas 4.2 taking into account the zero error, sample displacement, instrumental broadening, lattice constant and size- and strain-induced Gaussian-/Lorentzian peak broadening. Additionally, a single peak analysis was carried out using MDI Jade 9.

For transmission electron microscopy (TEM), Cu grids with a lacey carbon layer and 300 mesh from Plano $\mathrm{GmbH}$ were coated with the dispersed catalyst. The measurements were performed on a FEI Tecnai $\mathrm{G}^{2} 20$ S-TWIN transmission electron microscope with $\mathrm{LaB}_{6}$ cathode operating at an accelerating voltage of $200 \mathrm{kV}$.

Scanning transmission electron microscopy (STEM) was performed using a FEI Titan 80-200 ("ChemiSTEM") electron microscope with a Cs-probe corrector (CEOS GmbH) and a highangle annular dark field (HAADF) detector. ${ }^{13}$ The microscope was operated at $200 \mathrm{kV}$. In order to achieve " $Z$-contrast" conditions, a probe semi-angle of $25 \mathrm{mrad}$ and an inner collection semi-angle of the detector of $88 \mathrm{mrad}$ were used. Compositional maps were obtained with energy-dispersive $\mathrm{X}$ ray (EDX) spectroscopy using four large-solid-angle symmetrical Si drift detectors. For EDX elemental mapping, Pt L and Ni $\mathrm{K}$ peaks were used. The error of the EDX composition measurement is \pm 2 at $\%$.

\section{Electrochemical characterization}

The electrochemical characterization of the catalysts was performed in a three-compartment electrochemical glass cell filled with $0.1 \mathrm{M} \mathrm{HClO}_{4}$ solution as the electrolyte at room temperature. A Pt mesh and a $\mathrm{Hg} / \mathrm{Hg}_{2} \mathrm{SO}_{4}$ electrode were used as the 
counter electrode and reference electrode, respectively, and a commercial rotating disk electrode (RDE) setup from Pine Instruments equipped with a glassy carbon disk $(5 \mathrm{~mm}$ in diameter) was used. All measurements were controlled using a VSP-5 potentiostat from BioLogic.

Catalyst inks were prepared by dispersing about $5 \mathrm{mg}$ of the catalyst powder in $3.98 \mathrm{~mL}$ of Milli-Q water, $1 \mathrm{~mL}$ of 2-propanol and $20 \mu \mathrm{L}$ of Nafion solution (5 wt $\%$ ). The inks were homogeneously dispersed by ultrasonication. To prepare a thin catalytic film with about $8 \mu \mathrm{g}_{\mathrm{Pt}} \mathrm{cm}^{-2}$ on the GC electrode surface, $10 \mu \mathrm{L}$ of the prepared ink was drop-cast and the electrode was dried at $60{ }^{\circ} \mathrm{C}$ for $7 \mathrm{~min}$ in air. Cyclic voltammograms were collected in deaerated $0.1 \mathrm{M} \mathrm{HClO}_{4}$ electrolyte under a nitrogen atmosphere in the voltage range between +0.05 and $+1.00 \mathrm{~V}_{\mathrm{RHE}}$. For activation, 25 cycles were performed with a scan rate of $100 \mathrm{mV} \mathrm{s}^{-1}$. The ECSA was obtained using the mean integral charge of the underpotentially deposited hydrogen area as well the desorption area corrected for the capacitive current and Pt pseudo-capacity of $210 \mu \mathrm{C} \mathrm{cm}_{\mathrm{Pt}}{ }^{-2}$, assuming that one hydrogen atom observed correlates to one platinum atom. For ORR measurements, linear sweep voltammetry (LSV) was performed in an oxygenated $0.1 \mathrm{M} \mathrm{HClO}_{4}$ electrolyte solution. The polarization curves were recorded by anodic sweeping from +0.05 to $+1.00 \mathrm{~V}_{\mathrm{RHE}}$ with a scan rate of $5 \mathrm{mV} \mathrm{s}^{-1}$ and a rotation speed of $1600 \mathrm{rpm}$. The intrinsic kinetic current at $+0.9 \mathrm{~V}_{\mathrm{RHE}}$ was corrected for the mass transport diffusion limiting current between +0.2 and $+0.6 \mathrm{~V}_{\mathrm{RHE}}$.

Stability measurements were performed via potential cycling - two times, 4000 cycles - between +0.5 and $+1.0 \mathrm{~V}_{\mathrm{RHE}}$ with a scan rate of $50 \mathrm{mV} \mathrm{s}^{-1}$ in $\mathrm{N}_{2}$-saturated $0.1 \mathrm{M} \mathrm{HClO}_{4}$ at $0 \mathrm{rpm}$. Before and after each stability protocol, three CVs between +0.05 and $+1.0 \mathrm{~V}_{\mathrm{RHE}}$ at $100 \mathrm{mV} \mathrm{s}^{-1}$ were recorded. ORR activity was measured after activation (25 CVs), after 4000 cycles and after subsequent 4000 ones, i.e. 8000 cycles, of each electrode, using the same conditions as those described above.

\section{Results and discussion}

\section{Structural and morphological transformations during thermal annealing}

Bulk structural analysis. PtNi octahedral shaped nanoparticles with the nominal composition $\mathrm{PtNi}_{1.5}$ were prepared using a solvothermal, autoclave based synthesis procedure. The unannealed PtNi alloy catalyst (henceforth referred to as "asprepared") with $8 \mathrm{~nm}$ mean particle size was subjected to postsynthesis thermal annealing in a hydrogen flow (4 vol\% $\mathrm{H}_{2} / \mathrm{Ar}$ mixtures) at temperatures between 200 and $500{ }^{\circ} \mathrm{C}$. The resulting catalysts are henceforth labeled " $\mathrm{H}_{2} \mathrm{~T}$ ", with $T$ being their respective annealing temperature. For all obtained catalyst samples, inductively coupled plasma-optical emission spectroscopy (ICP-OES) analysis revealed no change in the actual atomic Pt : Ni composition of 38 at\% Pt and 62 at\% Ni or in the actual Pt weight loading on the carbon support (20 wt\% Pt) (see Table S1 (ESI $\dagger$ ) for details).

The long-range crystalline structure of the catalysts after annealing was investigated by X-ray diffraction (XRD), and the patterns are depicted in Fig. 1a. All samples exhibited patterns typical of the face centered cubic (fcc) structure type. The comparison with the reflections of pure Pt fcc (ICSD 4-0802) and pure Ni fcc (ICSD 4-0850) evidenced the formation of PtNi alloys. Furthermore, the reflections of the as-prepared PtNi are rather broad and asymmetric with a tailing on the low-angle side. With increasing annealing temperature, a gradual change in the lattice structure occurred: reflections shifted to higher angles (see gray dashed lines in Fig. 1a). In addition, all peaks became more symmetric, best visible for the (220) reflection. As the elemental composition remained constant, the reflection shift directly correlates with lattice structural changes in the bimetallic nano-octahedra.

Rietveld refinement of each pattern revealed details of the structural changes upon annealing. The best refinement results were obtained using a single fcc disordered PtNi alloy phase, which was modified by interstitial oxygen atoms on the vacant tetrahedral sites with refined occupancy. The influence of the high surface area carbon support, especially near the alloy (111) reflection (Fig. S1, ESI $\dagger$ ), was carefully subtracted and corrected for. Accordingly, the Rietveld analysis evidenced phase-pure PtNi alloy nanoparticles with some residual lattice oxygen atoms. Further details of the refinement as well as the detailed values of the structural parameters are given in the ESI on page 2 and Table $\mathrm{S} 2, \dagger$ and two refinement deconvolutions are presented in Fig. S2 (ESI $\dagger$ ). In Fig. 1b-d, the Rietveld-derived experimental lattice constant $a$, the microstrain $e_{0}$, and the mean crystallite size are plotted against annealing temperature. Consistent with simplified single peak analyses (see Fig. S3, ESI $\dagger$ ), gradual lattice contraction was evident above $200{ }^{\circ} \mathrm{C}$. The microstrain $e_{0}$, also referred to as raw microstrain, was obtained from Rietveld refinement without any processing. It is generally understood as a measure for local disorder in atomic lattices and is derived from the variation of the lattice parameter around the mean, i.e. influencing the peak width without a change in its position. Typically, the microstrain decreases with increasing crystallite size. ${ }^{\mathbf{1 4 - 1 6}}$ Herein, the evolution of the microstrain displayed a maximum for the $\mathrm{H}_{2} 300{ }^{\circ} \mathrm{C}$ catalyst, while the mean crystallite size (structural coherence length) increased monotonically with annealing temperature starting from the as-prepared sample. Thus, up to $300{ }^{\circ} \mathrm{C}$ annealing temperature, the microstrain and coherence length of the nanooctahedra exhibited an unusual positive correlation; in other words, the nanoparticles of $\mathrm{H}_{2} 200{ }^{\circ} \mathrm{C}$ and $\mathrm{H}_{2} 300{ }^{\circ} \mathrm{C}$ exhibited more local disorder than that expected from their size. This excess microstrain and its origin will become the subject of our discussion of activity trends below. Starting from $300{ }^{\circ} \mathrm{C}$, the microstrain and crystallite size follow their usual inverse relationships (Fig. S4, ESI $\dagger$ ). Both the lattice constant and microstrain reached a minimum for the PtNi-500 sample, suggesting a structurally and energetically relaxed alloyed state of the PtNi nanoparticles. Looking at the crystallite size, we can conclude that an increase in the coherence length occurred by annealing at $500{ }^{\circ} \mathrm{C}$, while $\mathrm{Pt}$ and $\mathrm{Ni}$ interdiffusion removed elemental anisotropies leading to more homogeneously alloyed lattices.

Particle ensemble morphology. To cross-correlate the X-ray diffraction derived mean crystallite size with the actual mean nanoparticle size and track morphological changes upon annealing, all samples were investigated by (Scanning) 

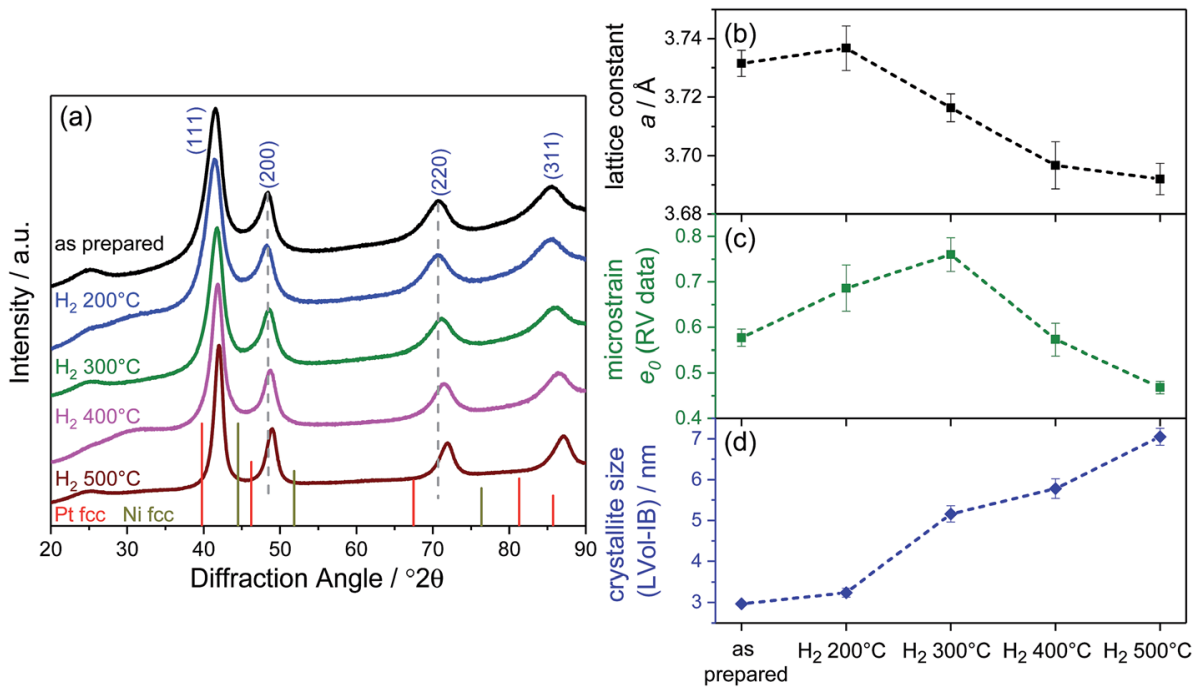

Fig. 1 X-ray diffraction study of the as-prepared and annealed samples: (a) measured XRD patterns, where red and yellow bars indicate pure metals Pt fCC (ICSD 4-0802) and Ni fCC (ICSD 4-0850). Dashed lines are a guide to the eye for the peak shift to higher angles with increasing temperature. (b-d) Structural impact of annealing temperature as derived from Rietveld refinement: (b) lattice constant, (c) microstrain and (d) crystallite size. Dashed lines are a guide to the eye.

Transmission Electron Microscopy, (S)TEM. Fig. 2A shows the bright-field (BF) TEM image of the as-prepared PtNi nanoparticle ensemble. Its largely octahedral morphology remained stable to thermal annealing in hydrogen up to $300{ }^{\circ} \mathrm{C}$ (Fig. 2C) beyond which major morphological transformations set in. This $300{ }^{\circ} \mathrm{C}$ temperature threshold was also supported by highangle annular dark field (HAADF) STEM analysis results in
Fig. 3K and $\mathrm{L}\left(300^{\circ} \mathrm{C}\right)$ and Fig. $3 \mathrm{P}$ and $\mathrm{Q}\left(400{ }^{\circ} \mathrm{C}\right)$. Annealing at $400{ }^{\circ} \mathrm{C}$ caused the octahedral particles to transform into a cuboctahedral shape, which is mainly composed of $\{111\}$ and $\{100\}$ facets (Fig. 3Q). As a consequence, the particles appeared rounded in the low magnification images (Fig. 2D and 3P). At $500{ }^{\circ} \mathrm{C}$, the octahedral shape was irreversibly lost and nearspherical particles were observed (Fig. 2E).
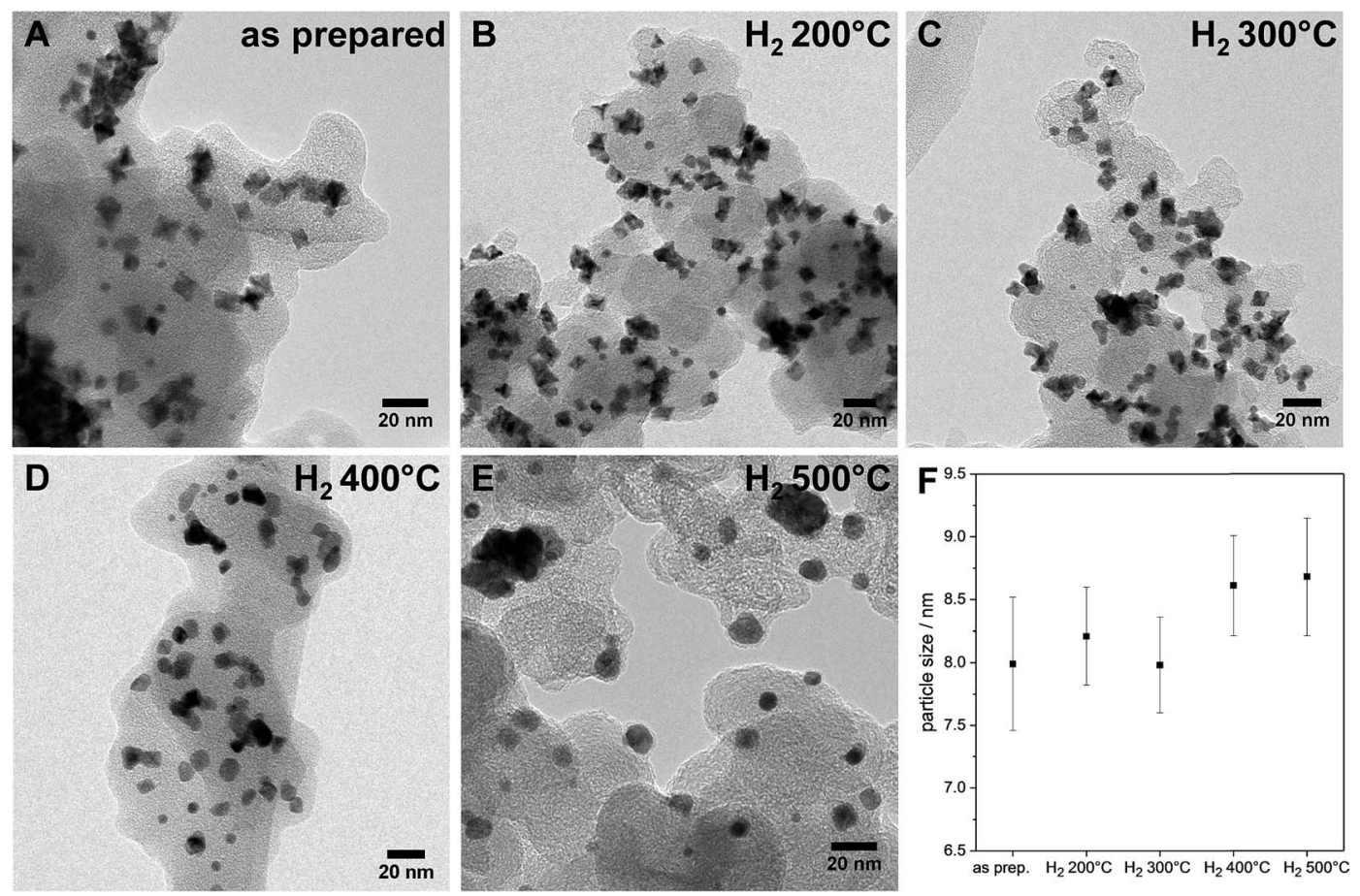

Fig. 2 (A-E) Evolution of the morphology upon annealing in hydrogen: TEM micrographs (BF) of various PtNi nanoparticles of the as-prepared sample and samples annealed at different temperatures; (F) particle sizes with a dependence on annealing temperature - error bars represent the calculated error of each particle size (details of the calculation given in ESI, $\uparrow$ page 6). 


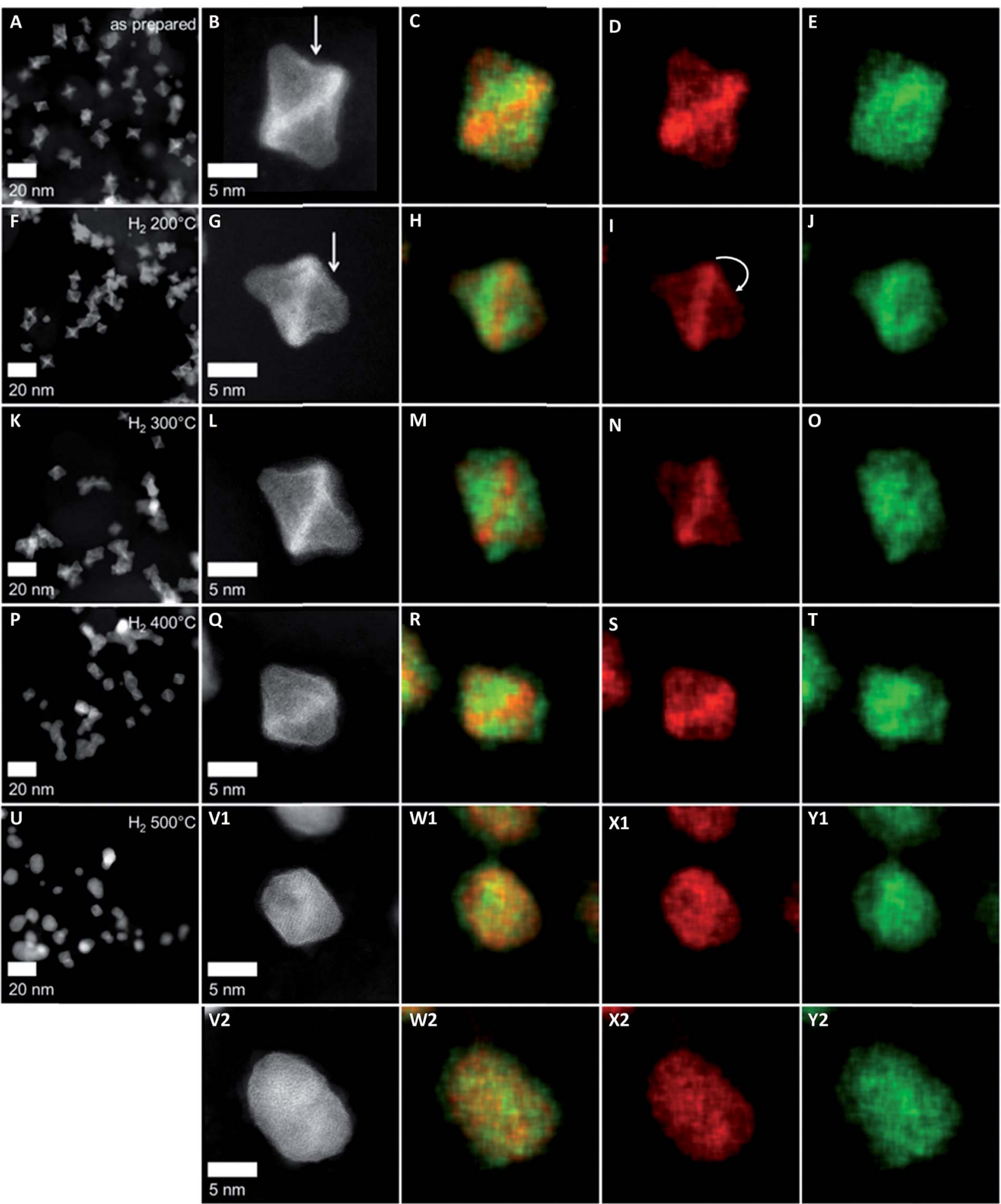

Fig. 3 HAADF STEM images ( $Z$ contrast) and EDX elemental maps of shaped PtNi nanoparticles without (A-E) and after annealing in hydrogen at different temperatures $(F-T)-(F-J) 200^{\circ} \mathrm{C},(K-O) 300^{\circ} \mathrm{C},(\mathrm{P}-\mathrm{T}) 400^{\circ} \mathrm{C}$ and $(\mathrm{U}-\mathrm{Y}) 500^{\circ} \mathrm{C}:(\mathrm{A}, \mathrm{F}, \mathrm{K}, \mathrm{P}$ and $\mathrm{U})$ low magnification overview HAADF STEM images of the nanoparticle ensembles; ( $B, G, L, Q$ and $V$ ) high resolution HAADF STEM images of shaped PtNi nanoparticles oriented close to a $\langle 110\rangle$ zone axis; ( $C-E, H-J, M-O, R-T$, and $\mathrm{W}-\mathrm{Y})$ EDX elemental maps of the corresponding nanoparticles; Pt and $\mathrm{Ni}$ are colored in red and green, respectively.

Interestingly, upon annealing, the TEM-based particle size remained essentially constant (Fig. 2F), despite the severe morphological changes after annealing to $500{ }^{\circ} \mathrm{C}$ - the particle size distributions are depicted in Fig. S5 (ESI†). Only a few coalesced particles were formed after annealing at $400{ }^{\circ} \mathrm{C}$ and $500{ }^{\circ} \mathrm{C}$, which were presumably closely neighboring particles before annealing. The generally maintained particle size explains largely localized Pt atom diffusion and redistribution 
on each particle. Furthermore, the stark discrepancy between the TEM-derived mean particle size and the XRD-derived crystallite size of the PtNi domains (Fig. 1d) likely suggests the presence of multi-grain particles at least up to $300{ }^{\circ} \mathrm{C}$. With higher annealing temperature, the variation of the elemental distribution results in growth and Ni enrichment of the PtNi domains and, thereby, leads to the structural relaxation observed for $\mathrm{H}_{2} 500{ }^{\circ} \mathrm{C}$.

Elemental anisotropy and particle concavity. To learn more about the local elemental Pt or Ni enrichment and segregation inside the nano-octahedra, STEM-EDX elemental maps were recorded (Fig. 3). The initial as-prepared PtNi nanoparticles appear concave in the $Z$ contrast (Fig. 3A and B). The combined EDX map of such octahedral nanoparticles oriented close to a $\langle 110\rangle$ zone axis (Fig. 3C) indicated the enrichment of Ni in the $\{111\}$ facets and a Pt-rich frame, evident by a red Pt-rich strip across the center of the nanoparticles. Accordingly, the nanoparticles constitute imperfect Pt octahedra with concave $\{111\}$ facets (Fig. 3D). Hence, in line with previous work, ${ }^{17,18}$ these asprepared octahedral nanoparticles feature an anisotropic elemental distribution of Pt and $\mathrm{Ni}$ atoms. We note that the concave shape in the HAADF STEM images of the as-prepared catalyst (Fig. 3A and $\mathrm{B}$ ) primarily represents the $\mathrm{Pt}$ atom distribution (Fig. 3D), due to the much weaker Z-contrast of $\mathrm{Ni}$ atoms (we will term this structural feature "Pt-concave" hereafter). However, since segregated $\mathrm{Ni}$ atoms "fill" the $\{111\}$ concave cavities of the particles (Fig. 3E), the facets of the asprepared particles are actual smooth, strongly Ni-enriched ones. Compositional quantification of the as-prepared NPs yielded an average composition of Pt 43 at\% and Ni 57 at\%, which is in reasonable agreement with the sample-averaged ICP-OES value of $\mathrm{Pt} 37$ at\% and Ni 63 at\%, considering possible surface dissolution of $\mathrm{Ni}$ atoms during the time the NPs were kept in the dispersion prior to the STEM-EDX investigations. The annealed nanoparticles showed an even better agreement between STEM-EDX and ICP-OES compositions (see Table S1, ESI $\dagger$ ) suggesting a stabilizing effect of annealinginduced $\mathrm{Pt}$ and $\mathrm{Ni}$ interdiffusion against $\mathrm{Ni}$ dissolution.

The NPs of $\mathrm{H}_{2} 200{ }^{\circ} \mathrm{C}$ showed only slight morphological changes (Fig. 3F) compared to the as-prepared sample, where some individual Pt-concave $\{111\}$ facets (arrow in Fig. 3B) appeared to transform into somewhat less concave $\{111\}$ facets (arrow in Fig. 3G). The corresponding EDX elemental maps suggested the migration of Pt atoms from the corner to the initially Ni-enriched $\{111\}$ facets (arrow in Fig. 3I), while the distribution of $\mathrm{Ni}$ is similar to that in the as-prepared state (see Fig. 3J). Accordingly, thermal annealing at moderate temperatures appears to heal Pt-concave $\{111\}$ facets. ${ }^{19}$ At the same time, the rearrangement of $\mathrm{Pt}$ from vertices to facets is associated with interdiffusion of $\mathrm{Pt}$ and $\mathrm{Ni}$ atoms in the $\{111\}$ facets.

The NPs of $\mathrm{H}_{2} 300{ }^{\circ} \mathrm{C}$ retained their octahedral shape with a Pt distribution similar to that of $\mathrm{H}_{2} 200{ }^{\circ} \mathrm{C}$ (Fig. $3 \mathrm{~L}$ and $\mathrm{N}$ ), having distinct Pt-concave $\{111\}$ facets. Fig. $3 \mathrm{O}$ shows that $\mathrm{Ni}$ atoms, likely in the oxidized chemical state, can segregate to the NP surface and form adatom layers on the $\{111\}$ facets of the octahedron. This confirms the findings of Ahmadi et al. ${ }^{20}$ who demonstrated preferential $\mathrm{NiO}_{x}$ surface segregation by moderate thermal annealing $\left(300{ }^{\circ} \mathrm{C}\right)$. We believe that the observed elemental distribution at $300{ }^{\circ} \mathrm{C}$ induced by the $\mathrm{Pt}$ and $\mathrm{Ni}$ interdiffusion within the octahedra is responsible for the elevated values of local compositional and structural disorder, i.e. the microstrain (Fig. 1c).

As mentioned above, at $400{ }^{\circ} \mathrm{C}$, the octahedral shape finally gave way to a cuboctahedral one, identified by clearly visible $\{111\}$ and $\{100\}$ facets (Fig. 3Q). This is induced by the migration of Pt atoms from the vertices to the $\{111\}$ facets, resulting in a structure which appears flat in the $Z$ contrast and hence, a smooth, flat Pt alloy surface. We observed similar transformations from octahedra to cuboctahedra during an in situ annealing TEM experiment. ${ }^{10}$ Based on the current data, we conclude that the morphological transformation from octahedra to cuboctahedra during heating is a thermodynamically driven effect rather than an effect of the gas atmosphere. This is also supported by the theoretical work of Wang et al. ${ }^{21}$ showing that cuboctahedra are energetically favorable over octahedra due to complete $\{100\}$ facet reconstruction. Furthermore, the morphological transformation to cuboctahedra and even more so the concomitant Pt and Ni intermixing are accompanied by a decrease of the lattice constant and microstrain as well as by a larger crystallite size of the PtNi domains (Fig. 1).

Finally, the octahedral shape was completely lost after annealing at $500{ }^{\circ} \mathrm{C}$ with only very few NPs remaining in the cuboctahedral shape (Fig. 3V1). These remaining cuboctahedra showed a more homogeneous distribution of the elements compared to all other samples. The former Pt-rich strip across the center of the cuboctahedral NPs is no longer visible in the elemental map (Fig. 3W1). Irregularly shaped NPs like those in Fig. 3V2, which potentially expose higher-index facets, are now found more frequently than cuboctahedral NPs. Furthermore, a homogeneous Pt and Ni distribution and a thin surface Ni layer characterize such NPs, as shown in exemplary EDX composition maps in Fig. 3W2-Y2. The observation of a more homogeneously alloyed particle structure in $\mathrm{H}_{2} 500{ }^{\circ} \mathrm{C}$ is in full accordance with the trends in our XRD-derived structural descriptors such as low microstrain, contracted lattice parameters and the largest crystallite size.

The atomic-scale morphological changes offer a plausible explanation for the crystallite size-microstrain correlations: the strong decrease in local disorder in the PtNi-400 and PtNi-500 NPs (Fig. 1c) can be attributed to the evolution of octahedral particles into cuboctahedral particles with reduced concave surface curvature. The initial concave morphology, coupled with Pt and Ni interdiffusion and Ni segregation, is the source of local disorder and accounts for elevated values of microstrain in the particle ensemble.

\section{Effect of local disorder and facet morphology on electrocatalytic ORR activity and stability}

Catalytic ORR activity and particle facet morphology. To link the structure and facet morphology to catalytic ORR activity, all catalysts were tested in a three-compartment cell using the thinfilm rotating disk electrode (TF-RDE) technique. Prior to ORR testing, the nano-octahedra were electrochemically dealloyed, 
i.e. leaching of surface $\mathrm{Ni}$ atoms into the electrolyte, and, thereby, catalytically activated using electrochemical cycling in $\mathrm{N}_{2}$-saturated $\mathrm{HClO}_{4}(0.1 \mathrm{M})$ in the potential range from +0.05 to $+1 \mathrm{~V}_{\mathrm{RHE}}\left(100 \mathrm{mV} \mathrm{s}^{-1}\right)$. The corresponding voltammograms (CVs) of cycle 25 are depicted in Fig. 4a. All samples displayed voltammetric features typical for Pt alloy facets: broad hydrogen ad-/desorption peaks $\left(+0.06\right.$ to $\left.+0.4 \mathrm{~V}_{\mathrm{RHE}}\right)$, double layer capacitive currents $\left(+0.4\right.$ to $\left.+0.6 \mathrm{~V}_{\mathrm{RHE}}\right)$, and Pt hydroxide and oxide peaks $\left(+0.7\right.$ to $\left.+1 \mathrm{~V}_{\mathrm{RHE}}\right)$. The electrochemically active surface area based on the hydrogen underpotential deposition charge $\left(\mathrm{H}_{\text {upd }}\right)$ from Fig. 4 a remained stable up to $300{ }^{\circ} \mathrm{C}$ (ECSA $\sim 40 \mathrm{~m}^{2} \mathrm{~g}_{\mathrm{Pt}}{ }^{-1}$ ) and dropped for the samples $\mathrm{H}_{2} 400{ }^{\circ} \mathrm{C}$ and $\mathrm{H}_{2}$ $500{ }^{\circ} \mathrm{C}\left(\right.$ ECSA $\left.\sim 30 / 25 \mathrm{~m}^{2} \mathrm{~g}_{\mathrm{Pt}}{ }^{-1}\right)$. More detailed information about the ECSA data is given in Fig. S6 (ESI $\dagger$ ). Since after annealing only a slight increase in the particle size was detected by TEM (Fig. 2F), we conclude that the morphological transformations as well as the Pt diffusion occurring above $300{ }^{\circ} \mathrm{C}$ (Fig. 2 and 3) must have influenced the ECSA of the dealloyed particles, with Pt-concave octahedral NPs exhibiting a larger ECSA than well-alloyed cuboctahedral or spherical ones. This is plausible and can be rationalized as follows: after performing the voltammetric activation/dealloying cycles, all segregated pure Ni surface atoms are assumed to be leached away, and the surfaces of the octahedral particles can be safely described to be significantly Ni depleted. Accordingly, well-alloyed cuboctahedral PtNi particles retain a larger atomic ratio of Ni atoms near the surface after activation/dealloying compared to the Ptconcave $\{111\}$ octahedra having segregated flat $\mathrm{Ni}\{111\}$ facets before activation. As hydrogen adsorption is energetically less favorable on Ni-rich alloy surfaces, ${ }^{4,22}$ the resulting $\mathrm{H}_{\mathrm{upd}}$ charge is depressed for the samples $\mathrm{H}_{2} 400{ }^{\circ} \mathrm{C}$ and $\mathrm{H}_{2} 500{ }^{\circ} \mathrm{C}$. In view of the experimentally observed facet morphology, we can conclude that the morphology of the activated/dealloyed catalyst particles follow the Pt-concave morphology of the annealed particles. In other words, the as-prepared, $\mathrm{H}_{2} 200{ }^{\circ} \mathrm{C}$ and $\mathrm{H}_{2} 300^{\circ} \mathrm{C}$ samples exhibit concave Pt-rich $\{111\}$ facets, while the $\mathrm{H}_{2} 400{ }^{\circ} \mathrm{C}$ and $\mathrm{H}_{2}$ $500{ }^{\circ} \mathrm{C}$ samples feature flat Ni-richer $\{111\}$ facets after activation/dealloying (also see Fig. 6 for more details).

The electrocatalytic Pt-based mass activity for the reduction of molecular oxygen to water was measured by anodic linear sweep voltammetry (LSV). Detailed experimental LSV curves are shown in Fig. S7 (ESI $\dagger$ ). Fig. 4b shows a single maximum for $\mathrm{H}_{2}$ $300{ }^{\circ} \mathrm{C}$ in the Pt-mass activity values at $+0.9 \mathrm{~V}_{\mathrm{RHE}}$ of the PtNi nanoparticles as a function of their annealing temperature. The ORR activity sharply decreased with the onset of the morphological transformations of facets and particles from octahedral
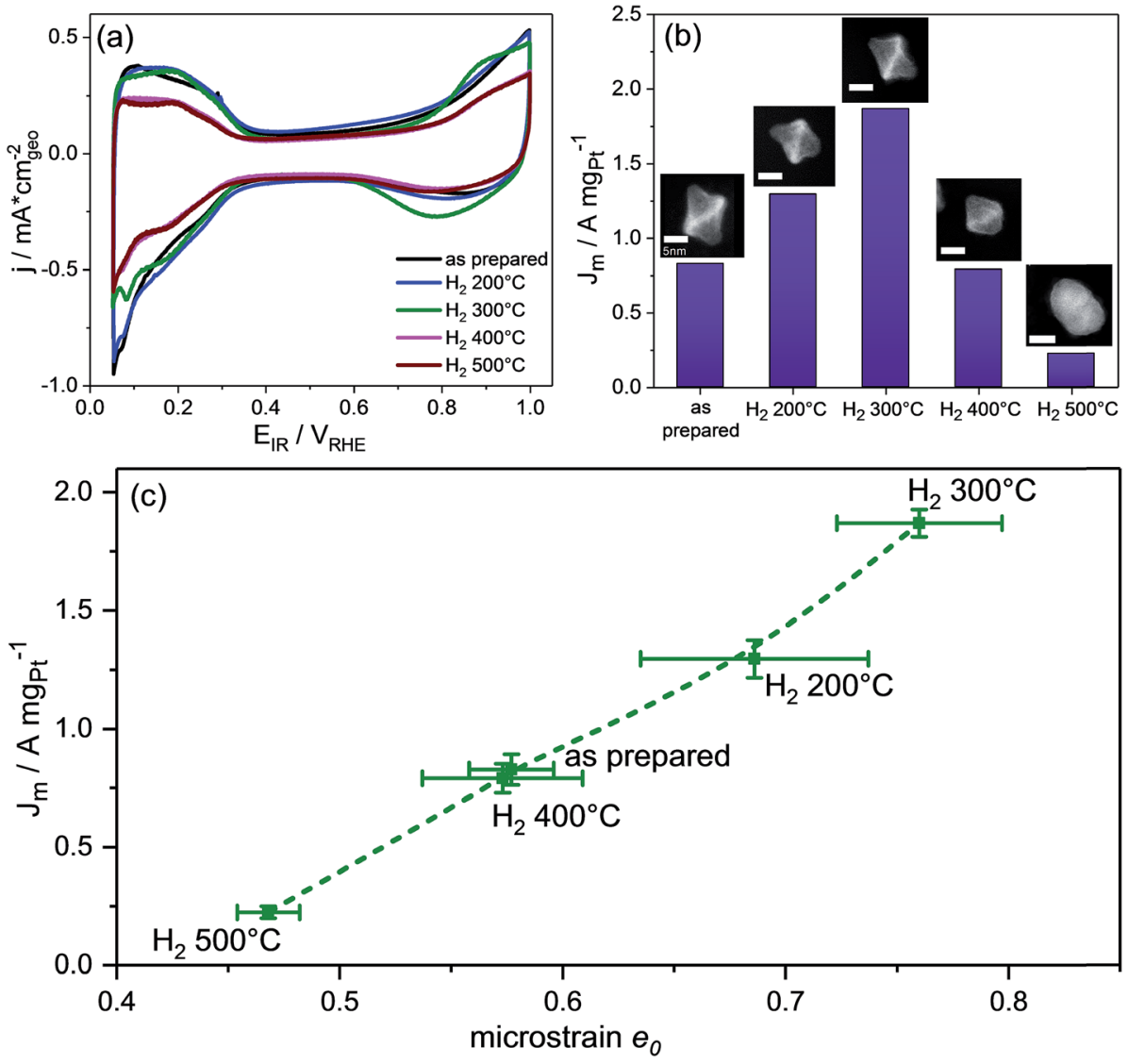

Fig. 4 Correlation of the structure and catalytic ORR activity for shaped PtNi nanoparticles: (a) cyclic voltammograms of the 25th cycle of activation in a $\mathrm{N}_{2}$-saturated electrolyte in the potential range from +0.05 to $+1 \mathrm{~V}_{\mathrm{RHE}}$ with $100 \mathrm{mV} \mathrm{s}^{-1}$. (b) Pt mass-based ORR activity, $\mathrm{J}_{\mathrm{m}}$, evaluated at $+0.9 \mathrm{~V}_{\mathrm{RHE}}$ during an anodic voltammetric scan in an $\mathrm{O}_{2}$-saturated electrolyte in the potential range from +0.05 to $+1 \mathrm{~V}_{\mathrm{RHE}}$ with $5 \mathrm{mV} \mathrm{s}^{-1}$ at $1600 \mathrm{rpm}$. The potentials are IR corrected, and $0.1 \mathrm{M} \mathrm{HClO}_{4}$ was used as the electrolyte. (c) Correlation of microstrain and ORR activity. Sample names are indicated at each data point; the green dashed line represents the proposed trend and acts as a guide to the eye. 


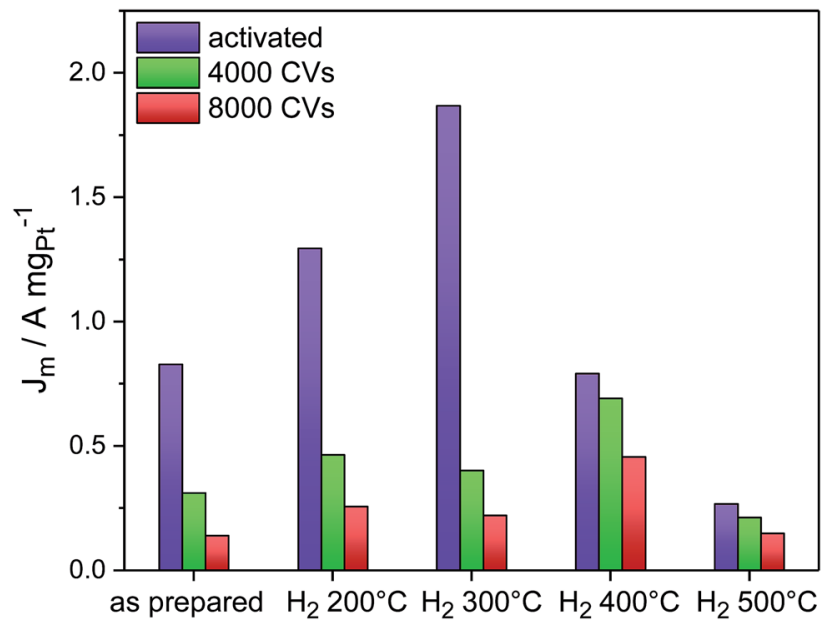

Fig. 5 Performance stability of shaped PtNi nano-catalysts as a function of their annealing temperature: Pt mass activity, $J_{m}$, for the electrochemical ORR after 4000 (green) and 8000 (brown) potential cycles. The "activated" value (purple) is the ORR activity after 25 activation cycles (+0.05 to +1.0 $\mathrm{V}_{\text {RHE, }} 100 \mathrm{mV} \mathrm{s}^{-1}$ ) in $\mathrm{N}_{2}$. Conditions: oxygen transport-corrected kinetic $J_{m}$ values were evaluated at $+0.9 \mathrm{~V}_{\mathrm{RHE}}$ in an $\mathrm{O}_{2}$-saturated electrolyte with anodic scans in the potential range from +0.05 to $+1 \mathrm{~V}_{\mathrm{RHE}}$ with $5 \mathrm{mV} \mathrm{s}^{-1}$ at $1600 \mathrm{rpm}$. Stability cycling was performed in a $\mathrm{N}_{2}$-saturated electrolyte in the potential range from +0.5 to $+1.0 \mathrm{~V}_{\mathrm{RHE}}\left(50 \mathrm{mV} \mathrm{s}^{-1}\right)$. The potentials are IR corrected, and $0.1 \mathrm{M} \mathrm{HClO}_{4}$ was used as the electrolyte.

with Pt-concave $\{111\}$ facets to cuboctahedral with nearly flat facets to spherical with potentially higher-index facets. Taking into account the very similar particle size and the fact that cuboctahedra are confined by $\{111\}$ and $\{100\}$ facets, the lower activity of the catalyst $\mathrm{H}_{2} 400{ }^{\circ} \mathrm{C}$ is in full agreement with earlier structure sensitivity studies that reported $\mathrm{Pt}_{3} \mathrm{Ni}\{111\}$ surfaces to be superior to $\mathrm{Pt}_{3} \mathrm{Ni}\{100\}$ surfaces. ${ }^{4}$ Anyway, we note that the catalyst $\mathrm{H}_{2} 400{ }^{\circ} \mathrm{C}$ shows a similar activity as the as-prepared sample with Pt-concave $\{111\}$ facets. Furthermore, our data confirmed that the complete loss of octahedral shape coupled to interdiffusion and surface alloying of $\mathrm{Pt}$ and $\mathrm{Ni}$ atoms $\left(\mathrm{H}_{2} 500{ }^{\circ} \mathrm{C}\right)$ is very detrimental for the ORR activity.

Linking catalytic activity and local disorder. We compared the trends in the catalytic ORR activity of PtNi nano-octahedra with those of their structural parameters derived from diffraction analysis whose results are presented in Fig. 1b-d. In doing so, we uncovered a strong correlation between the catalytic activity and lattice microstrain. Fig. 4c illustrates the close relationship (green dashed line as a guide to the eye) between the experimental ORR activity and the degree of local lattice structural and lattice compositional disorder, the microstrain, of the PtNi nanoparticle ensembles. We prove once more that microstrain appears to be a suitable experimentally accessible predictor for catalytically ORR active Pt alloy nanomaterials as also reported earlier ${ }^{23}$ - at least within the present family of PtNi alloys. The activity-disorder trend is further confirmed by the similar values of the as-prepared sample and the catalyst $\mathrm{H}_{2}$ $400{ }^{\circ} \mathrm{C}$. The structural and morphological transformations and relaxations of $\mathrm{H}_{2} 400{ }^{\circ} \mathrm{C}$ (see Fig. 1 and 2D) appear to nearly offset each other with respect to the resulting bulk lattice microstrain. Smooth, flat Pt alloy $\{111\}$ and $\{100\}$ facets with a more homogeneous elemental $\mathrm{Pt}$ and $\mathrm{Ni}$ atom distribution in the cuboctahedral NPs of $\mathrm{H}_{2} 400{ }^{\circ} \mathrm{C}$ (Fig. 2 and 3) yield a similar level of local disorder as the Pt-concave $\{111\}$ facets in the octahedral NPs of the as-prepared catalyst.

The previous discussion of the catalytically most active structural state of the activated $300{ }^{\circ} \mathrm{C}$ annealed PtNi nano-octahedra suggests an intrinsic link between the experimental facet morphology and the sample-averaged microstrain caused by chemical and lattice disorder. In fact, DFT-calculations of the binding energies of reaction intermediates of the ORR on $\mathrm{Pt}$ nanofacets with concave curvature have recently revealed that over-coordinated Pt atoms prevalent on surfaces with concave characteristics display lower thermodynamic limiting potentials. $^{\text {24,25 }}$ Invoking arguments from the Brønsted-Evans-Polanyi (BEP) relationships between thermodynamic driving forces and activation energies of elementary steps, smaller thermochemical overpotentials imply a lower kinetic barrier of the rate determining step, and, thus, improve catalytic ORR rates. We argue that, roughened by the Ni-depleting activation/dealloying step, the resulting concave particle facets display a large number of such catalytically active over-coordinated surface Pt atoms. At the same time, we argue that roughened concave particle facets with their inhomogeneous distribution of Pt and Ni display a level of lattice and compositional disorder, which exceeds that of well-alloyed flat cuboctahedra or well-alloyed spherical particles. Hence, the former nanoparticles are more likely to exhibit large values of $e_{0} \cdot{ }^{12}$

Morphological and performance stability. A major challenge related to the fuel cell operation of Pt-based bimetallic ORR cathode electrocatalysts is their performance stability during extended potential cycling, where both the composition and morphology affect their performance. Following the activity trends, the influence of the annealing temperature on the catalyst performance stability was investigated during prolonged potential cycling. Two consecutive sets of 4000 cycles each were applied, and the ECSA and ORR activity values were recorded after activation/dealloying, after 4000 cycles and after 8000 cycles. The corresponding CVs and LSVs are presented in Fig. S8 and S9 (ESI $\dagger$ ), respectively.

Fig. 5 shows the evolution of the Pt mass-based ORR activity of the annealed catalysts during the cycling test protocol. A monotonic decrease in activity was observed for the as-prepared catalyst as well as the catalysts $\mathrm{H}_{2} 200{ }^{\circ} \mathrm{C}$ and $\mathrm{H}_{2} 300{ }^{\circ} \mathrm{C}$. The activity of all three samples converged despite major differences in their initial structural states. The activity degradation is attributed to leaching of Ni from the particle facets. In fact, continued electrochemical dissolution of $\mathrm{Ni}$ atoms from below the surface, even from initially Pt-enriched facets, is known to lower the ORR activity. ${ }^{17}$ The ECSA values of the three catalysts decreased as well (Fig. S10, ESI $\dagger$ ), but not proportional to the Pt mass activity, indicating that surface modifications alone cannot account for performance losses. Looking at the other two high temperature catalysts, it is evident that annealing of PtNi nano-octahedra to $400{ }^{\circ} \mathrm{C}$ resulted in improved performance stability. Consistently, the ECSA of $\mathrm{H}_{2} 400{ }^{\circ} \mathrm{C}$ remained very stable over 8000 cycles $\left(\sim 30 \mathrm{~m}^{2} \mathrm{~g}^{-1}\right)$. The initially low activity of $\mathrm{H}_{2} 500{ }^{\circ} \mathrm{C}$ remained at a very low level over $8000 \mathrm{CVs}$. 


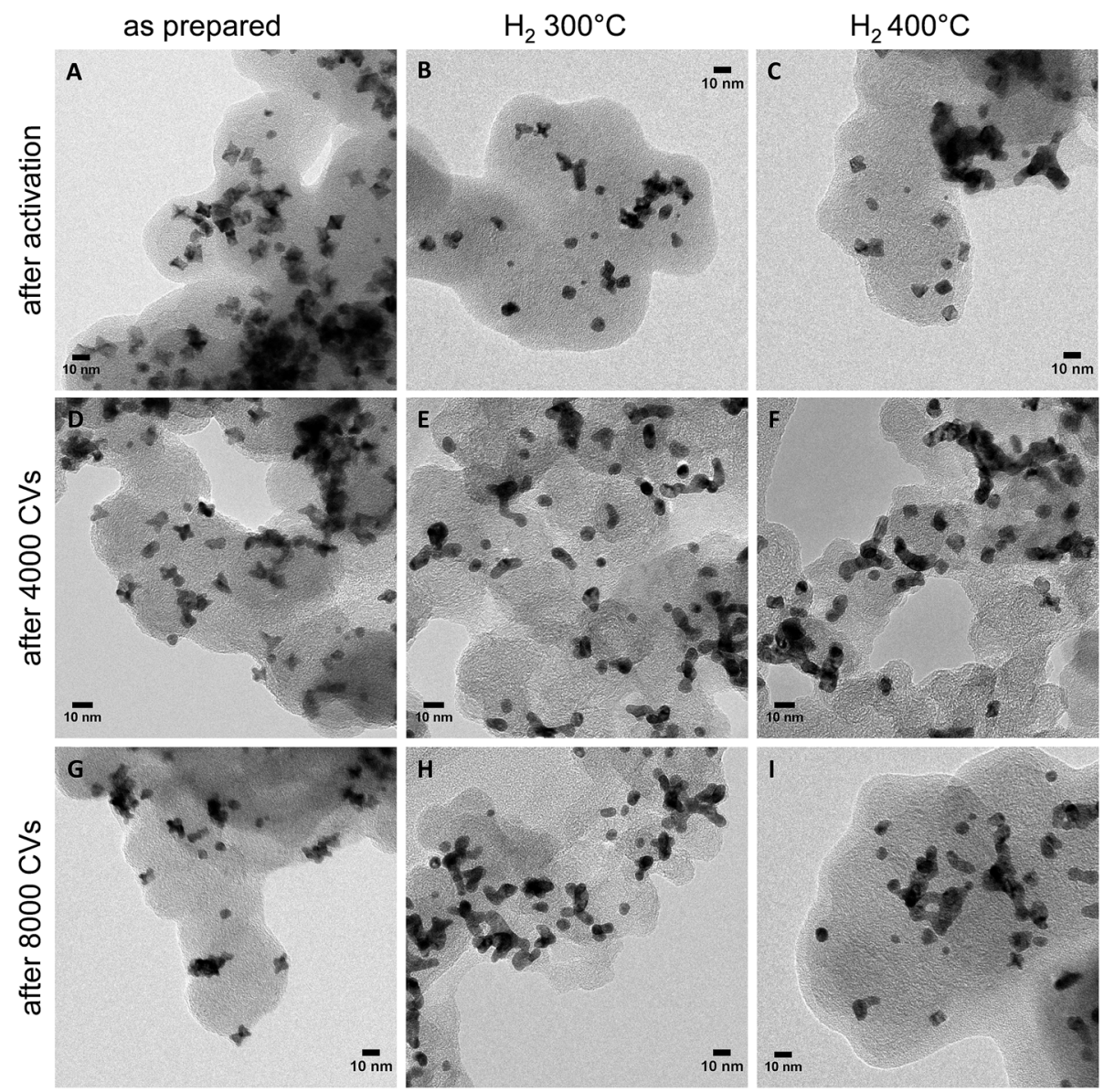

Fig. 6 Morphological stability of shaped PtNi nanoparticles: TEM micrographs of samples without and with annealing after electrochemical cycling: (A-C) after activation (25 CVs), (D-F) after 4000 cycles, and (G-I) after 8000 cycles. (A, D and G) the as-prepared PtNi octahedra, (B, E and $\mathrm{H}) \mathrm{H}_{2} 300^{\circ} \mathrm{C}$ catalyst sample, (C, F and I) $\mathrm{H}_{2} 400{ }^{\circ} \mathrm{C}$ catalyst sample.

Imperfect $\{111\}$ facets with Pt-concave curvature appear to be a recipe for high initial ORR activity, yet a reasonable balance between activity and stability is achieved by a morphological transformation from concave octahedral to a facet-healed cuboctahedral shape via thermal annealing. On comparing all catalysts together, it is found that the catalyst $\mathrm{H}_{2} 400{ }^{\circ} \mathrm{C}$ maintained the highest Pt mass ORR activity after 8000 cycles, and, as shown by STEM images (Fig. 3P and Q), it is characterized by cuboctahedral particles. This conclusion is in good agreement with a recent computational study by Mahata et al. ${ }^{26}$ which proposed cuboctahedral Pt NCs to be electrochemically more stable than concave or convex octahedral Pt nanoparticles.

Morphological stability is a major challenge for shapecontrolled alloy nanocatalysts, as it directly controls the evolution of the surface area and intermediate adsorption via structure sensitivity effects, and, hence, plays a crucial role in the understanding of the empirical catalytic performance stability. This is why morphological changes of the PtNi nano-catalysts were investigated by TEM after activation as well as after 4000 and 8000 potential cycles during stability tests. TEM micrographs of the as-prepared, $\mathrm{H}_{2} 300^{\circ} \mathrm{C}$ and $\mathrm{H}_{2} 400^{\circ} \mathrm{C}$ nanoparticle catalysts are shown in Fig. 6. Additional images of $\mathrm{H}_{2} 200{ }^{\circ} \mathrm{C}$ after activation and of $\mathrm{H}_{2} 500{ }^{\circ} \mathrm{C}$ after activation and 8000 cycles are provided in Fig. S11 (ESI $\dagger$ ).

After activation, the as-prepared catalyst (Fig. 6, left column) exhibited nano-octahedra with strongly concave $\{111\}$ facets (Fig. 6A). Their particle surface morphology apparently converged to their initial Pt-based STEM morphology. We attribute this to its characteristic anisotropic elemental distribution (Fig. 3B and D): Ni atoms were leached from the flat $\mathrm{Ni}$ segregated $\{111\}$ facets and, thus, gave way to the observed excavated concave facets with a Pt-enriched surface. After $\geq 4000$ potential cycles, octahedra with further excavated facets, and hexapod-shaped NPs emerged, due to prolonged Ni leaching from the surfaces. In fact, compositional analysis confirmed that the Pt: Ni ratio increased from $40: 60$ before electrochemical cycling to $93: 7$ (EDX) after cycling. Thus, only 14 at\% Ni were left after 4000 cycles and 7 at $\%$ after 8000 cycles, and this explains the severe loss in ORR activity. A similar mechanism was observed for the catalysts $\mathrm{H}_{2} 200^{\circ} \mathrm{C}$ (Fig. S11A, ESI $\dagger$ ) and $\mathrm{H}_{2} 300^{\circ} \mathrm{C}$ (Fig. 6, middle column): initial dealloying and $\mathrm{Ni}$ dissolution resulted in concave and excavated octahedral NPs. A significantly distinct behavior, however, was noted for $\mathrm{H}_{2} 300^{\circ} \mathrm{C}$ after 4000 cycles, where neither excavated octahedral NPs or any form of hexapod-shaped NPs were left behind. The particles 
were either rounded or were attached by their facets to form particle agglomerated clusters. The degradation continued after 8000 cycles and resulted in irregularly shaped clusters.

A distinct degradation behavior was displayed by the catalyst $\mathrm{H}_{2} 400{ }^{\circ} \mathrm{C}$ (Fig. 6, right column). This material preserved its alloyed cuboctahedral particle shape after activation. Surprisingly, cuboctahedra were partially present even after 8000 cycles. Partial cluster formation by facet attachment of neighboring particles was prevalent, as well. In fact, the shape stability of the NPs of $\mathrm{H}_{2} 400{ }^{\circ} \mathrm{C}$ is accompanied by a low degree of $\mathrm{Ni}$ dissolution as determined by EDX of several catalyst agglomerates. 41 at\% Ni are left after 4000 cycles and 37 at $\% \mathrm{Ni}$ after 8000 cycles, whereas $\mathrm{H}_{2} 300{ }^{\circ} \mathrm{C}$ contains only 19 at\% $\mathrm{Ni}$ after 4000 and 8000 cycles. See detailed compositional stability data after electrochemical cycling in Table S3 (ESI $\dagger$ ). In the NPs of $\mathrm{H}_{2} 500{ }^{\circ} \mathrm{C}$, no significant morphological changes were observed after electrochemical cycling (Fig. S11B and C, ESI $\dagger$ ).

These morphological observations can plausibly account for the experimental performance stability trajectories. The activated concave octahedral particles of $\mathrm{H}_{2} 300{ }^{\circ} \mathrm{C}$ with their enhanced local disorder (microstrain) showed the highest initial ORR activity, and the activated cuboctahedral NPs with flat $\{111\}$ and $\{100\}$ facets or spherical ones showed lower initial ORR activity. As the NPs continued to get depleted near the surface due to the prolonged electrochemical cycling and once they started to lose their concave octahedral shape by transforming into Pt-rich hexapods (Fig. 6D and G) or aggregated clusters (Fig. 6E and H), their initial high ORR activity dropped. On the other hand, the well-alloyed cuboctahedral particles annealed at $400{ }^{\circ} \mathrm{C}$ were able to retain their $\mathrm{Ni}$ content to a larger degree and appeared morphologically more resistant to potential cycling (Fig. 6F and I), showing only little change in the shape as well as ORR activity after 8000 cycles.

\section{Conclusions}

We set out to explore whether PtNi nano-octahedra with rather ordered, smooth alloy facets, or nano-octahedra with rather imperfect, disordered, concave facets are more active and stable ORR electrocatalysts, and which structural parameter controls and possibly predicts their activity. To check this, we have investigated the relationship between some bulk structural parameters, the individual particle morphology and the catalytic ORR activity of a family of annealed octahedral-shaped PtNi nanoparticles.

We found that the post-synthetic annealing procedures between 200 and $500{ }^{\circ} \mathrm{C}$ transformed the as-prepared nanooctahedra stepwise into faceted cuboctahedra and finally into rather spherical nanoparticles with a mix of potentially higherindex facets. We have identified the experimentally accessible sample-averaged microstrain, a measure of local disorder in crystalline phases, as the best predictor and descriptor for the electrochemical ORR activity of such nano-octahedra.

Nano-octahedra with high microstrain and concave Pt curvature catalytically outperformed cuboctahedra with flat, well-alloyed $\{111\}$ and $\{100\}$ nanofacets. More specifically, concave nano-octahedra with enhanced local compositional and structural disorder (microstrain) were invariably superior to more ordered and well-alloyed PtNi nanoparticles. Accordingly, our results describe a new structural feature beneficial for ORR activity deviating from the conventional concept of well-ordered and well-alloyed $\mathrm{Pt}_{3} \mathrm{Ni}(111)$ skin surface as the mechanistic origin for enhanced experimental ORR activities in nanooctahedra.

While catalytically active, the concave PtNi octahedra lacked sufficient morphological stability, lost excessive amount of $\mathrm{Ni}$ and transformed into Ni poor hexapods, which is why cuboctahedral shaped PtNi nanoparticles prepared at $400{ }^{\circ} \mathrm{C}$ ultimately offered the most favorable balance between ORR activity, performance stability, and morphological stability. Accordingly, such nanoparticles are proposed as promising future PEM cathode fuel cell catalysts.

\section{Conflicts of interest}

There are no conflicts to declare.

\section{Acknowledgements}

We acknowledge financial support given by the Deutsche Forschungsgemeinschaft (DFG) grant STR 596/5-1 \& 596/5-2 ("Shaped Pt bimetallics") and HE 7192/1-1 \& 7192/1-2 as well as by the German Ministry of Education and Research (BMBF) via the project "LoPlaKats" (number 03SF0527A).

\section{References}

1 M. D. Macia, J. M. Campina, E. Herrero and J. M. Feliu, J. Electroanal. Chem., 2004, 564, 141-150.

2 A. Kuzume, E. Herrero and J. M. Feliu, J. Electroanal. Chem., 2007, 599, 333-343.

3 N. M. Marković and P. N. Ross, Surf. Sci. Rep., 2002, 45, 117229.

4 V. R. Stamenkovic, B. Fowler, B. S. Mun, G. F. Wang, P. N. Ross, C. A. Lucas and N. M. Markovic, Science, 2007, 315, 493-497.

5 J. B. Wu, J. L. Zhang, Z. M. Peng, S. C. Yang, F. T. Wagner and H. Yang, J. Am. Chem. Soc., 2010, 132, 4984-4985.

6 J. Zhang, H. Yang, J. Fang and S. Zou, Nano Lett., 2010, 10, 638-644.

7 J. B. Wu, A. Gross and H. Yang, Nano Lett., 2011, 11, 798-802. 8 M. K. Carpenter, T. E. Moylan, R. S. Kukreja, M. H. Atwan and M. M. Tessema, J. Am. Chem. Soc., 2012, 134, 8535-8542.

9 C. Cui, L. Gan, H.-H. Li, S.-H. Yu, M. Heggen and P. Strasser, Nano Lett., 2012, 12, 5885-5889.

10 M. Gocyla, S. Kuehl, M. Shviro, H. Heyen, S. Selve, R. E. Dunin-Borkowski, M. Heggen and P. Strasser, ACS Nano, 2018, 12, 5306-5311.

11 P. Strasser, Science, 2015, 349, 379-380.

12 R. Chattot, O. Le Bacq, V. Beermann, S. Kuhl, J. Herranz, S. Henning, L. Kuhn, T. Asset, L. Guetaz, G. Renou, J. Drnec, P. Bordet, A. Pasturel, A. Eychmuller, T. J. Schmidt, P. Strasser, L. Dubau and F. Maillard, Nat. Mater., 2018, 17, 827-833. 
13 A. Kovács, R. Schierholz, K. Tillmann, Ernst Ruska-Centre for Microscopy and Spectroscopy with Electrons (ER-C), et al., FEI Titan G2 80-200 CREWLEY, Journal of LargeScale Research Facilities, 2016, 2, A43, DOI: 10.17815/jlsrf17812-17868.

14 P. S. V. Mocherla, C. Karthik, R. Ubic, M. S. Ramachandra Rao and C. Sudakar, Appl. Phys. Lett., 2013, 103, 022910.

15 W. Qin, T. Nagase, Y. Umakoshi and J. A. Szpunar, Philos. Mag. Lett., 2008, 88, 169-179.

16 K. Maniammal, G. Madhu and V. Biju, Physica E, 2017, 85, 214-222.

17 C. Cui, L. Gan, M. Heggen, S. Rudi and P. Strasser, Nat. Mater., 2013, 12, 765-771.

18 L. Gan, C. Cui, M. Heggen, F. Dionigi, S. Rudi and P. Strasser, Science, 2014, 346, 1502-1506.

19 L. Gan, M. Heggen, C. Cui and P. Strasser, ACS Catal., 2016, 6, 692-695.
20 M. Ahmadi, F. Behafarid, C. Cui, P. Strasser and B. Roldan Cuenya, ACS Nano, 2013, 7, 9195-9204.

21 G. Wang, M. A. Van Hove, P. N. Ross and M. I. Baskes, J. Chem. Phys., 2005, 122, 024706.

22 S. Rudi, X. Tuaev and P. Strasser, Electrocatalysis, 2012, 3, 265-273.

23 R. Chattot, T. Asset, P. Bordet, J. Drnec, L. Dubau and F. Maillard, ACS Catal., 2017, 7, 398-408.

24 F. Calle-Vallejo, J. Tymoczko, V. Colic, Q. H. Vu, M. D. Pohl, K. Morgenstern, D. Loffreda, P. Sautet, W. Schuhmann and A. S. Bandarenka, Science, 2015, 350, 185-189.

25 F. Calle-Vallejo, M. D. Pohl, D. Reinisch, D. Loffreda, P. Sautet and A. S. Bandarenka, Chem. Sci., 2017, 8, 22832289.

26 A. Mahata, K. S. Rawat, I. Choudhuri and B. Pathak, Catal. Sci. Technol., 2016, 6, 7913-7923. 